\title{
Variations de la croissance radiale de Quercus ilex $L$ en fonction du climat
}

\author{
SH Zhang, F Romane * \\ CNRS, centre Emberger, BP 5051, 34033 Montpellier cedex 1, France
}

(Reçu le 27 juiliet 1990; accepté le 27 novembre 1990)

\begin{abstract}
Résumé - La croissance radiale du chêne vert (Quercus ilex L), espèce dominante dans beaucoup de formations végétales du Bassin méditerranéen, est étudiée en relation avec les précipitations et les températures. L'analyse (analyses factorielles, procédure ARMA, etc) permet de montrer que l'existence des faux cernes, phénomène fréquent chez cette espèce, est favorisée par des précipitations estivales. L'épaisseur des cernes est aussi liée positivement aux précipitations estivales, alors que les températures des mois d'octobre à décembre précédant la saison de croissance sont liées négativement à cette épaisseur. Cette espèce est donc capable de profiter des précipitations estivales, alors qu'elle paraît "indifférente" aux conditions climatiques printanières, c'est-à-dire à celles de la phase principale de croissance.
\end{abstract}

cerne / climat méditerranéen / croissance radiale / préclpitations / Quercus ilex L / température

Summary - Diameter growth of Quercus ilex $L$ and the interannual variability of climatic characteristics. Diameter growth of Quercus ilex $L$, the dominant sclerophyllous species in much of the Mediterranean basin vegetation, was studied in relation to precipitation and temperature. The study site in Montpellier (Southern France) has a Mediterranean climate. Eleven trees, grown from acorns in 1963, were cut in 1984 and the rings studied at 3 levels (ground, 0.5 and $1 \mathrm{~m}$ above the ground). The data were analyzed by the ARMA procedure which gives the part of the total variation due to the autocorrelation processes. A multiple regression between the factors of a principal component analysis (PCA) of the monthly climatic data (rainfall and temperature) and the residuals issuing from the ARMA procedure then suggested that the large annual rings were due to a particular rainfall distribution during the year, the late summer precipitation promoting a large annual growth diameter (table II). The presence or absence of false rings (table l) was also analyzed by PCA (figs 2 and 3). The false rings were positively correlated to a large annual growth diameter corresponding to a high summer rainfall (fig 4), while the relationship with the temperature preceding the growing season was negative. It therefore appears that this species is relatively unaffected by spring climatic conditions, ie which generally indicate the main growth phase, but that it is able to take advantage of the interannual climatic variations of the region.

ring / Mediterranean climate / diameter growth / precipitation / Quercus ilex $L$ / temperature

\footnotetext{
* Adresse actuelle : Biotechnology Research Centre, Zhongshan Univ Guangzhov, 510272 PR China.
} 


\section{INTRODUCTION}

Le chêne vert (Quercus ilex L), espèce dominante de taillis qui constituent l'une des biocénoses les plus représentées dans le Bassin méditerranéen (Pons et Vernet, 1971; Quézel, 1985), apparaît comme une espèce valorisant souvent mal les milieux où elle se trouve. Plusieurs hypothèses peuvent être avancées pour tenter d'expliquer cette situation, telle celle d'un vieillissement exagéré des souches, dû à un très faible renouvellement par semis, ou encore, celle d'un épuisement du milieu édaphique dans une biocénose exploitée, pour ne pas dire surexploitée, depuis des millénaires.

L'une de ces causes pourraît être aussi le fait d'une mauvaise adaptation de cette espèce aux fortes variations climatiques interannuelles caractéristiques du climat méditerranéen; ainsi cette espèce tirerait mal parti des années favorables. C'est cet aspect qui sera analysé ici au travers de l'étude des variations de la croissance radiale. Dans le cas présent, n'ayant pas d'observations disponibles sur le stock d'eau dans le sol, nous faisons l'hypothèse que la liaison avec certains paramètres climatiques (précipitations, températures) peut constituer une première approche de cette question. Cette approche nous a par ailleurs conduits à nous pencher sur la question des faux cernes, assez nombreux chez cette espèce. En effet, la délimitation difficile des cernes chez le chêne vert (Venet, 1974) pose la question de savoir si un cerne correspond effectivement à une année ou s'il existe des "anomalies". L'hypothèse est faite que, si faux cernes il y a, ils sont liés à certaines conditions climatiques. Nous essaierons donc de dater ces faux cernes puis nous chercherons alors s'il existe des conditions climatiques particulières à cette date.

\section{SITE, MATÉRIEL ET MÉTHODE}

Le site expérimental, la station de Camp Redon, est situé dans le Sud de la France, sur le campus du Centre national de la recherche scientifique de Montpellier $\left(3^{\circ} 52^{\prime} \mathrm{N}, 43^{\circ} 38^{\prime} \mathrm{E}\right)$. II se trouve à une altitude de $50 \mathrm{~m}$, au sein d'un environnement karstique, dans une doline; le sol est apparenté au sol rouge fersiallitique méditerranéen, assez profond et caillouteux.

Les observations météorologiques ont été réalisées sur le site même; toutefois, pour certaines périodes d'observations manquantes, nous avons utilisé les données issues d'une station météorologique située à environ $2 \mathrm{~km}$ de distance, ce qui paraît raisonnable et possible dans ce domaine de la dendroclimatologie, car les variations en épaisseur des cernes sont essentiellement influencées par le macroclimat et la distance entre les arbres étudiés. La station météorologique peut donc être éloignée de quelques dizaines de $\mathrm{km}$ au maximum (Fritts, 1976).

Si l'on se réfère à l'analyse climatique de Debussche et Escarré (1983), cette station se trouve dans le climat méditerranéen subhumide au sens d'Emberger (1955), avec des précipitations moyennes annuelles de $857 \mathrm{~mm}$, une température moyenne pour les minima du mois le plus froid de $0,6^{\circ} \mathrm{C}$ et pour les maxima du mois le plus chaud de $28,7^{\circ} \mathrm{C}$ (période 1966-1979).

Ce site a été retenu car les observations ont pu y être faites sur un matériel dont nous connaissions l'âge, les individus de Quercus ilex L étudiés étant issus de semis réalisés en 1963. Dix arbres ont été coupés, ce qui de fait correspond à 11 échantillons en raison du dédoublement au ras du sol du tronc de l'un des arbres (le $n^{\circ} 6$ ). Sur chaque échantillon, 3 rondelles ont été découpées, après repérage de l'orientation, 1 à la base du tronc, 1 à $50 \mathrm{~cm}$ au-dessus du sol et 1 à $1 \mathrm{~m}$.

A partir de cernes caractéristiques permettant une interdatation (Wendland, 1975; Fritts, 1976; Serre, 1980), ainsi que par l'étude de la variation des dimensions des pores (vaisseaux), il a été possible de repérer les faux cernes (tableau 1), peu faciles à identifier chez cette espèce à cause des caractères anatomiques du bois (Venet, 1974, op cit). Puis la mesure de l'épaisseur des cernes a été effectuée, avec une précision de l'ordre de $10^{-2} \mathrm{~mm}$ en suivant, pour 
chaque rondelle, 3 rayons à $120^{\circ} \mathrm{C}$ perpendiculairement aux limites des cernes (Martin, 1974). Notons d'ailleurs que chez Quercus ilex, il est parfois préférable, ce qui a été fait ici, de suivre, non pas un rayon géométrique, mais un rayon ligneux parallèle à la direction de l'accroissement (Tessier, 1984). Si le rayon choisi passe par une zone "anormale" (départ de branche, par exemple), cette zone est évitée en suivant un autre rayon d'orientation un peu différente de celle du rayon originel.

Pour ce qui est de l'analyse des données, les facteurs influant sur l'épaisseur du cerne ont été étudiés par un modèle visant à cerner la part des variations due aux processus d'autocorrélation (Box et Jenkins, 1970; Guiot, 1981; Guiot et al, 1982). Le modèle mathématique retenu (procédure ARMA) est basé sur le fait que, dans une série chronologique, une part de la variation peut être expliquée, au moins en partie dans le cas général, par la liaison de chaque élément avec les éléments précédents, le reste de la variation étant alors considéré comme aléatoire, c'est-à-dire non expliqué par les processus d'autocorrélation :

$$
C_{t}=f\left(C_{t-1}, \ldots, C_{t-k}\right)+a_{t}
$$

où : $C_{t}=$ épaisseur du cerne l'année $t$,

$-f\left(C_{t-1}, \ldots C_{t-k}\right)$ est la fonction représentant la part du cerne de l'année $t$ dont la construction peut être expliquée par des cernes des années précédentes.

$-a_{t}=$ résidu aléatoire.

C'est ce résidu aléatoire que l'on tente alors d'expliquer par les facteurs climatiques. Dans ce but, une analyse en composantes principales a d'abord été effectuée sur les paramètres climatiques (variables), à savoir dans ce cas les 12 précipitations mensuelles $(P)$ avec, soit les 12 températures moyennes mensuelles $\left(T_{\text {moy }}\right)$, soit les 12 températures moyennes mensuelles des minimums journaliers $\left(T_{\min }\right)$, soit encore les 12 températures moyennes mensuelles des maximums journaliers $\left(T_{\max }\right)$. Dans cette analyse, les individus sont constitués par les années de la période analysée. Dans le cas, par exemple, des rondelles de la base dont la série porte sur 19 ans, le tableau pour chacune des analyses est donc de dimensions $19 \times 24$. Cette analyse en composantes principales a permis de dégager des facteurs principaux d'ordre climatique. L'explication du résidu aléatoire $a_{t}$, en fonction de ces facteurs principaux climatiques, a alors été faite grâce à une régression multiple (Guiot, 1981; Tessier, 1984; Zhang, 1987).

Pour les faux cernes, nous avons dans un premier temps cherché à établir une typologie des années avec faux cernes, ainsi que la caractérisation par les variables climatiques de cette typologie (Zhang, 1987). Dans ce but, une analyse en composantes principales a été réalisée, dans laquelle le premier ensemble (lignes ou individus) est celui des années d'observations et le deuxième ensemble (colonnes ou variables) celui de l'épaisseur des cernes annuels pour chacun des 11 échantillons, les variables climatiques (12 températures mensuelles et 12 précipitations mensuelles) étant prises comme variables supplémentaires. Seules les rondelles du niveau bas ont été utilisées pour cette analyse car fournissant les séries chronologiques de longueur maximale.

\section{RÉSULTATS}

\section{Croissance radiale et climat}

Notons d'abord qu'en général pour nos observations, la réponse de la croissance radiale aux facteurs de l'environnement est similaire quel que soit le niveau de la mesure. Ceci est illustré par la figure 1 où sont représentées les variations de l'épaisseur de tous les cernes aux 3 niveaux d'observation; il s'agit de courbes de synthèse, c'est-à-dire de courbes pour lesquelles les épaisseurs sont les moyennes pour tous les arbres et toutes les directions de mesure à un même niveau. Seuls les résultats pour le niveau de base sont donc présentés ici.

Pour l'analyse des variations de l'épais. seur des cernes (procédure ARMA), le résidu aléatoire a été expliqué par une régression multiple en fonction des facteurs principaux issus d'une analyse factorielle bâtie sur les 2 éléments du climat déjà évoqués (précipitations et températures). 


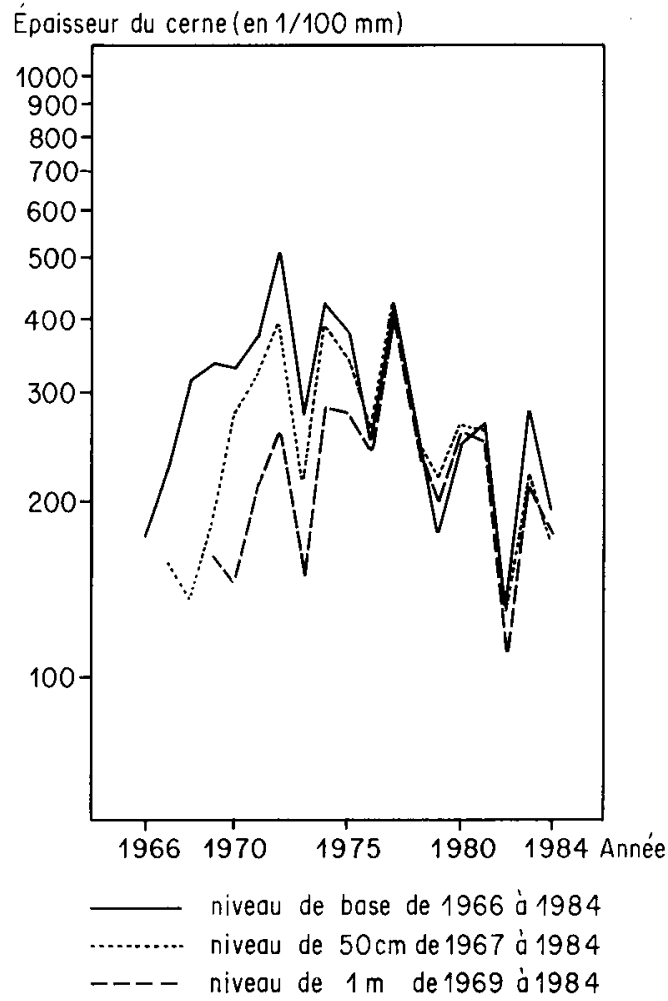

Fig 1. Courbes de synthèse de l'épaisseur des cernes aux 3 niveaux considérés. - niveau de base de 1966 à 1984; $\cdots$ niveau de $50 \mathrm{~cm}$ de 1967 à 1984; - - niveau de $1 \mathrm{~m}$ de 1969 à 1984.

Etant donné le petit nombre d'observations (ie 19 années) et la variabilité du climat dans la région étudiée (ie les décalages climatiques interannuels par rapport aux mois calendaires), it nous a paru judicieux d'utiliser, non pas les précipitations et les températures mensuelles, mais des regroupements de certains mois. Se pose alors la question du choix des combinaisons à faire. N'ayant pas de critères pour faire ce choix, nous avons analysé les données dans plusieurs cas de regroupements, déterminés empiriquement. Les résultats correspondant aux meilleures expli- cations du résidu aléatoire sont donnés dans le tableau II. De ce tableau il ressort que ce sont principalement les pluies d'été qui ont une influence positive sur l'épaisseur du cerne chez cette espèce. En revanche, les pluies du mois d'avril ont une influence négative sur la croissance.

Pour les températures, il se dégage moins facilement des tendances nettes, mais il ne faut pas oublier que les températures et les précipitations ne sont pas indépendantes. Ainsi les liaisons négatives durant les mois d'été peuvent s'expliquer probablement par le fait que des cernes correspondent à des étés pluvieux, qui sont aussi relativement plus frais que les autres.

\section{Présence d'un faux cerne et climat}

En se basant sur la mise en évidence de cernes caractéristiques, nos observations montrent qu'un cerne annuel est produit chaque année chez Quercus ilex et qu'il n'y a donc pas de cernes manquants; mais il peut $y$ avoir 1, parfois 2, faux cernes dans un cerne annuel, un faux cerne étant constitué par une bande de couleur plus sombre à l'intérieur d'un cerne annuel. La présence, ou l'absence, d'un faux cerne pour une année est en général constante le long du tronc, quelle que soit la hauteur.

Pour la période observée (1966-1984, soit 19 années), 6 années ne présentent pas de faux cerne et, sur les 13 en présentant, 2 années (1973 et 1976) n'en présentent que chez un seul individu (tableau I). L'analyse en composantes principales entre les années (premier ensemble) et les accroissements des cernes des 11 arbres (deuxième ensemble) fait nettement ressortir, dans le plan factoriel des axes I et II, les années pour lesquelles un faux cerne a été mis en évidence (fig 2), l'année avec faux cerne étant définie dans ce cas 
Tableau I. Présence des faux cernes de 1966 à 1984.

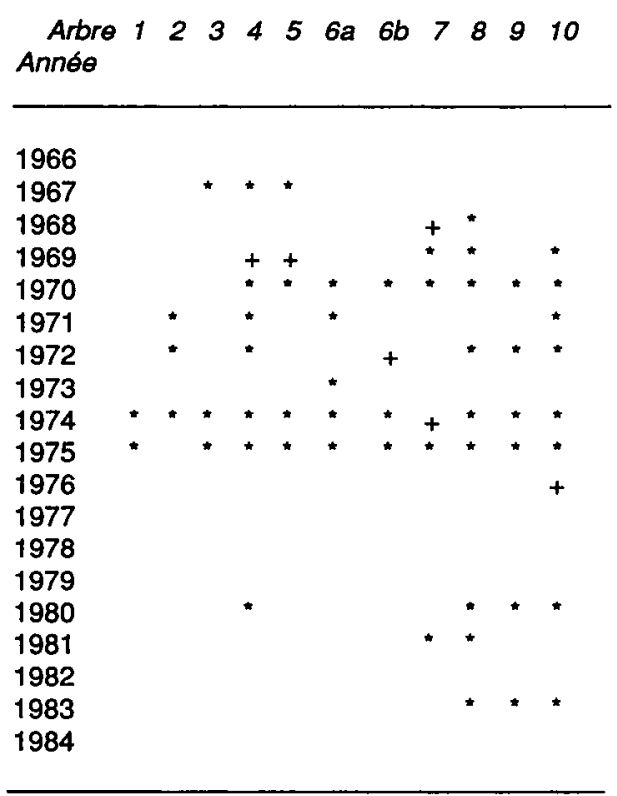

faux cerne peu visible (+) ou très visible (*). comme celle où il $y$ a eu un faux cerne pour au moins 2 arbres. Dans cette figure, l'axe I correspond, de la gauche vers la droite, à une diminution de la moyenne de l'épaisseur du cerne de tous les arbres dans une année. Ce diagramme fait nettement apparaître que les années avec un faux cerne sont généralement des années avec une croissance annuelle plus forte que celles sans faux cerne, sauf l'année 1977 où la croissance annuelle est forte mais sans faux cerne, cas particulier qui sera discuté ci-dessous. Pour cette même analyse en composantes principales, si c'est le deuxième ensemble, c'est-à-dire celui des accroissements des cernes des 11 arbres, qui est considéré (fig 3), les arbres $3,4,5,8$, et 9 sont des arbres qui produisent des cernes épais, quelle que soit l'année, les arbres 7 et 1 en produisant des minces et les arbres $2,6 a, 6 b$ et 10 correspondent à une épaisseur moyenne. Mais dans ce diagramme n'apparaît pas de liaison entre la croissance radiale des arbres et l'existence de faux cernes (les arbres ayant plus de 5 faux cernes sont indiqués par un astéristique dans cette fi-

Tableau II. Relation entre crissance en diamètre et plusieurs types de regroupements des précipitations et températures mensuelles (fonctions de réponse).

\begin{tabular}{|c|c|c|c|c|c|c|c|c|c|c|c|c|c|c|}
\hline \multirow[b]{2}{*}{ Regroupement } & \multicolumn{6}{|c|}{ Précipitations } & \multicolumn{6}{|c|}{ Températures } & \multirow[t]{2}{*}{$R^{2} C$} & \multirow[t]{2}{*}{$F$} \\
\hline & 0 & $N D$ & $j F m$ & $a$ & $M$ & JJAS & 0 & $N D$ & $j F m$ & $a$ & $M$ & JJAS & & \\
\hline $\begin{array}{l}P+T \operatorname{moy} \\
P+T \min \\
P+T \max \end{array}$ & $\begin{array}{l}1 \\
1 \\
1\end{array}$ & $\begin{array}{l}1 \\
1 \\
1\end{array}$ & $\begin{array}{l}1 \\
1 \\
1\end{array}$ & $\begin{array}{l}-2 \\
-2 \\
-2\end{array}$ & $\begin{array}{c}+1 \\
+2 \\
l\end{array}$ & $\begin{array}{l}+3 \\
+3 \\
+3\end{array}$ & $\begin{array}{l}-2 \\
-1 \\
-2\end{array}$ & $\begin{array}{r}-1 \\
1 \\
-2\end{array}$ & $\begin{array}{l}-2 \\
-1 \\
-2\end{array}$ & $\begin{array}{c}1 \\
+2 \\
-1\end{array}$ & $\begin{array}{l}1 \\
1 \\
1\end{array}$ & $\begin{array}{r}-2 \\
1 \\
-2\end{array}$ & $\begin{array}{l}0,93 \\
0,95 \\
0,91\end{array}$ & $\begin{array}{l}0,99 \\
0,99 \\
0,99\end{array}$ \\
\hline
\end{tabular}

Pour les regroupements : O, N, D : octobre à décembre de l'année $t-1 ; j, F, m, a, M, J, J, A, S:$ janvier à septembre de l'année $t$.

$P$ : précipitations; $T_{\text {moy }}$ : température moyenne; $T_{\min }$ : température moyenne minimale; $T_{\max }$ : température moyenne maximale

1, 2, 3 : niveaux de signification (respectivement supérieurs à $0,90,0,95$ et 0,99 ); le signe + ou - indique le sens de la corrélation (/ = non significatif)

$R^{2} C=$ coefficient de détermination; $F$ : signification globale (test F). 


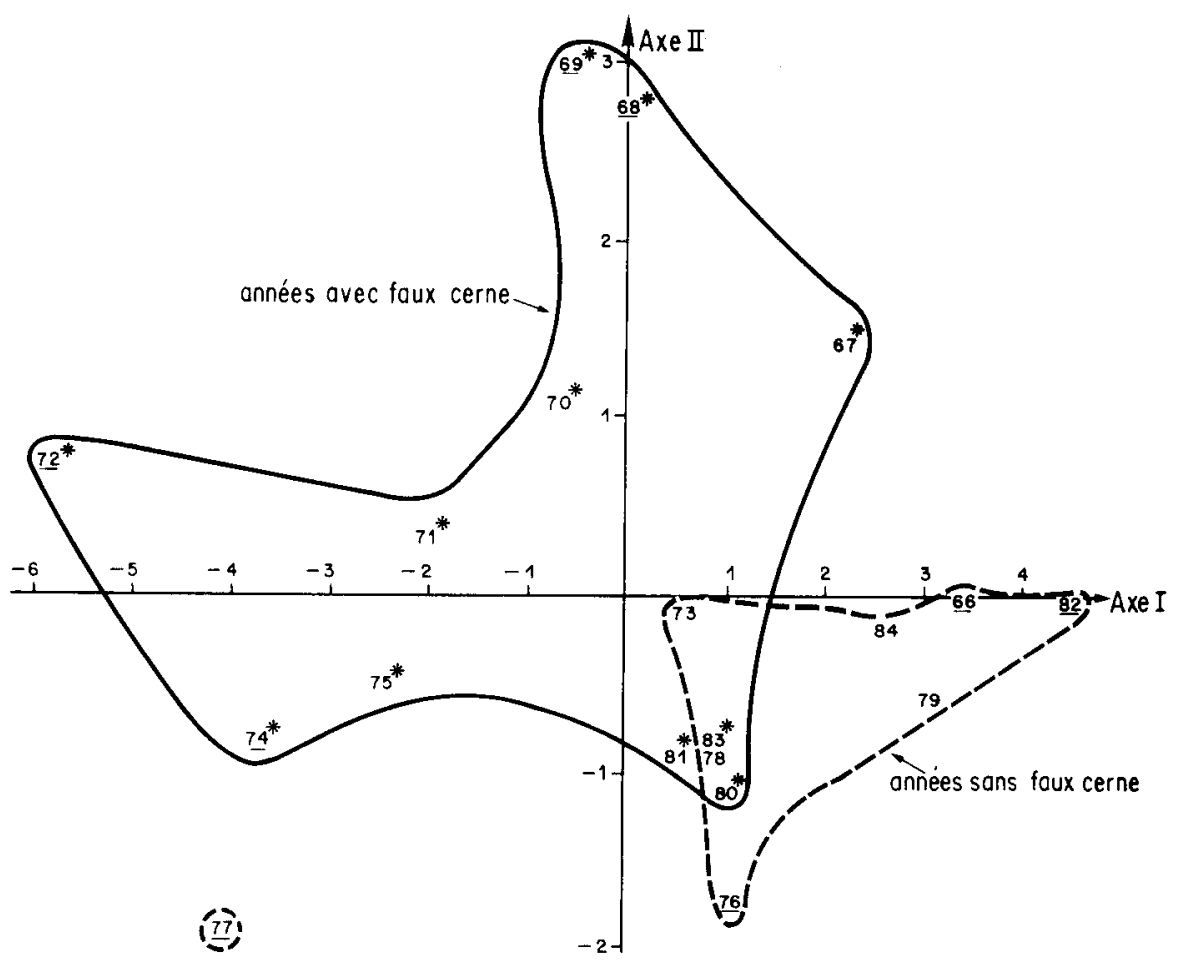

Fig 2. Analyse en composantes principales sur l'épaisseur annuelle des cernes. Projection sur le plan

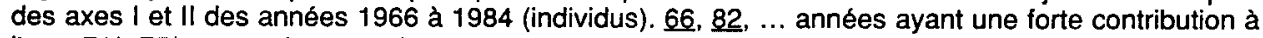
l'axe; $71^{*}, 75^{*}, \ldots$ années avec faux cerne.

gure 3). La production de faux cerne semble donc un phénomène plus lié aux conditions climatiques d'une année qu'à une prédisposition chez tel ou tel individu.

Dans le plan des axes I et II, nous avons rapporté (fig 3 ), comme variables supplémentaires, les précipitations et les températures moyennes mensuelles. La position particulière des précipitations du mois de septembre $\left(P_{g}\right)$ suggère que les pluies d'été jouent un rôle dans la formation de faux cernes. Dans une moindre mesure, interviennent aussi celles de juin $\left(P_{6}\right)$ qui peuvent être facilement assimilées

Tableau III. Précipitations mensuelles moyennes de 1964 à 1984.

Mois oct nov déc janv fév mars avr mai juin juil août sept




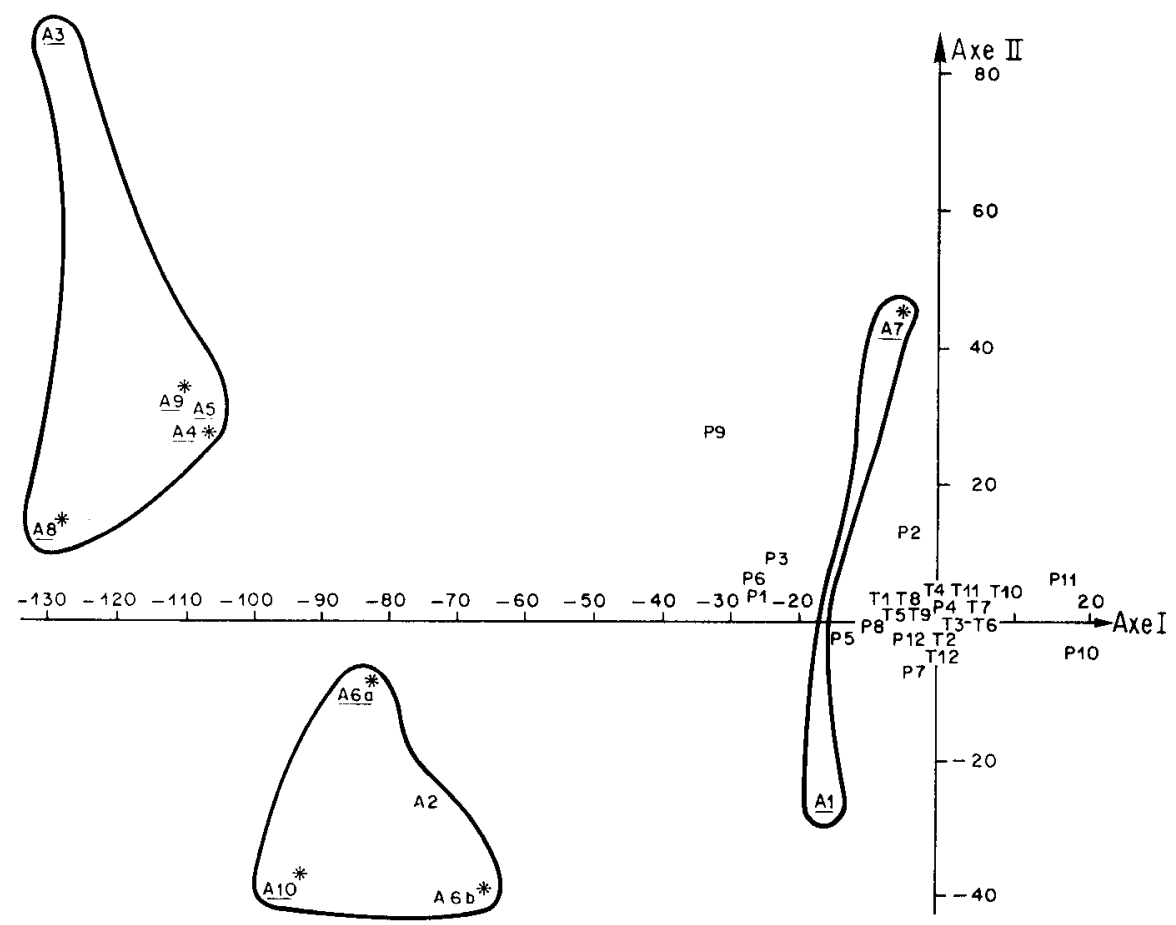

Fig 3. Analyse en composantes principales sur l'épaisseur annuelle des cernes. Projection sur le plan des axes I et II des arbres (variables) et des variables supplémentaires (précipitations et températures). $A_{1}$ à $A_{10}$ : arbres numérotés de 1 à $10 ; P_{1}$ à $P_{12}$ : précipitations des mois de janvier à décembre; $T_{1}$ à $T_{12}$ : températures moyennes des mois de janvier à décembre. $A 3$ : arbres ayant une forte contribution à l'axe; A6b*, ... arbres ayant de faux cernes pour au moins 5 années.

à des précipitations estivales et celles de mars $\left(P_{3}\right)$ et janvier $\left(P_{1}\right)$ pour lesquelles l'interprétation paraît plus difficile. Ceci est confirmé par la comparaison des pluies des mois d'été (juin-septembre, soit séparément, soit globalement) entre les années avec faux cerne et celles sans faux cerne (fig 4). Si ces différences vont toutes dans le même sens, elles ne sont toutefois statistiquement (test $t$ de Student) significatives que dans le cas du mois d'août $(P<$ 0,01 ). En revanche, pour la température moyenne mensuelle (pendant la période estivale), il n'y a pas de différence remar- quable entre les années avec un faux cerne et les années sans faux cerne (fig 4).

\section{DISCUSSION ET CONCLUSION}

L'un des caractères essentiels du climat méditerranéen auquel est soumis la station dans laquelle nos observations ont été faites est bien évidemment sa sécheresse estivale, sans oublier toutefois que nous sommes ici à la marge de cette zone climatique méditerranéenne (Aschmann, 


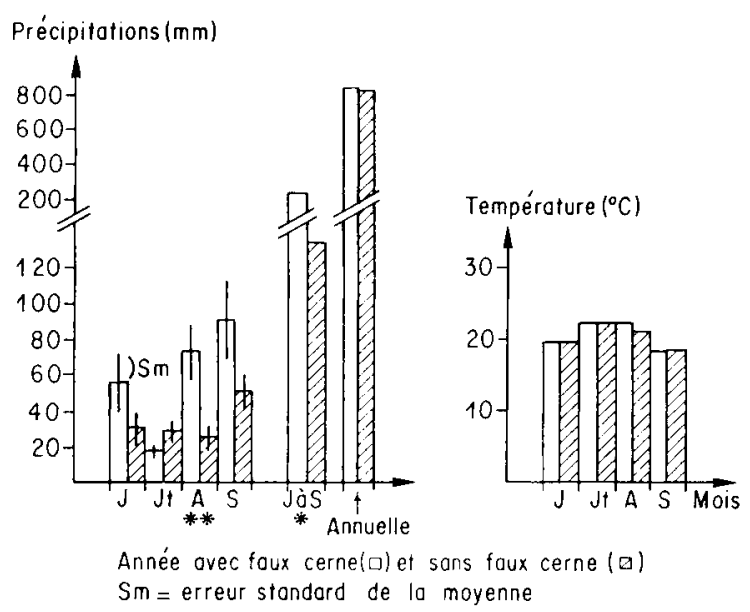

Fig 4. Précipitations et températures estivales des années avec faux cerne et de celles sans faux cerne. Année avec faux cerne (ㄱ) et sans faux cerne (E). $\mathrm{Sm}=$ erreur standard de la moyenne.

1973, 1984; Daget, 1980). Durant cette période estivale, l'eau est le principal facteur limitant de la croissance de la végétation (Aussenac et Valette, 1982). Bien que Quercus ilex puisse continuer les processus de transpiration et de photosynthèse dans un état de grande sécheresse (Eckardt, 1977; Aussenac et Granier, 1978; Aussenac et Valette, 1982), il n'en demeure pas moins que lorsque la plante a de la difficulté à absorber l'eau presque épuisée dans le sol, la photosynthèse peut s'arrêter à cause de la fermeture des stomates (Fritts, 1976; Aussenac et Valette, 1982). Ainsi une étude dans une autre station (Zhang, 1987) a montré qu'il y a une corrélation positive très significative entre la croissance radiale hebdomadaire du tronc de chêne vert et les précipitations de cette même semaine. Dans ces conditions, toute pluie estivale est donc utilisée, au moins partiellement, et cette croissance radiale vient se rajouter à une croissance printanière qui se fait de toute façon chaque année grâce aux précipitations, plus ou moins fortes certes, de cette période.
Nous avons cependant vu que les précipitations du mois d'avril jouent un rôle négatif, ce qui pourrait s'expliquer de 2 manières, différentes, mais nous manquons d'éléments pour étayer l'une ou l'autre de ces hypothèses.

II pourrait tout d'abord y avoir une action sur le système racinaire dont dépend l'utilisation de l'eau du sol (Aussenac et Valette, 1982), le rapport tige/racine diminuant en condition d'insuffisance en eau et en éléments minéraux (Bronwer, 1977). Si, en avril, début de la croissance pour Quercus ilex, le sol est pauvre en eau, il pourrait se produire un développement relativement plus fort des racines pour compenser la faiblesse de la réserve hydrique. Or le mois d'avril dans la station de Camp Redon a été un mois relativement sec (tableau (II), comparé aux mois de mars et de mai, pendant les 20 années d'observation (1964-1984). Ceci aurait donc entraîné un développement plus fort des racines, développement qui favorise par la suite l'utilisation de l'eau et donc une meilleure croissance radiale. Cette meilleure utilisation de 
l'eau disponible compenserait alors largement la faible (à cause du fort développement racinaire ce mois-là) croissance radiale du tronc durant le mois d'avril.

Une autre possibilité serait que les précipitations du mois d'avril favorisent la formation de jeunes pousses et les floraisons, ce qui aboutirait à une fructification plus abondante et donc à une dépense en substances de réserves plus importante, l'investissement d'énergie pour la croissance radiale étant alors réduit.

Les liaisons négatives avec les températures du mois d'octobre (tableau II), le mois le plus arrosé en moyenne dans la région de Montpellier (tableau III), peuvent s'interpréter ainsi : un mois d'octobre chaud l'année $t-1$ permettrait à la croissance radiale du tronc de se poursuivre entraînant ainsi l'utilisation de réserves qui ne seraient alors plus disponibles l'année $t$, d'où un effet dépressif sur la croissance de cette année $t$.

Une température élevée en été favorise l'évapo-transpiration, ce qui entraîne un épuisement plus rapide de l'eau. Une étude indépendante a montré aussi qu'il y a une corrélation négative très significative entre la variation hebdomadaire du diamètre du tronc du chêne vert et celle de la température maximale (Zhang, 1987; de la même semaine).

Quant au phénomène du faux cerne il est certainement lié dans le cas du chêne vert à sa croissance polycyclique (Champagnat, 1983a, 1983b) dans certaines conditions climatiques. La formation d'un faux cerne n'aurait lieu que dans le cas où il y a une diminution, ou même un arrêt momentané, de la croissance, suivi d'un nouveau départ de celle-ci. Ces conditions sont réalisées de fait assez souvent dans la région étudiée (climat de type méditerranéen atténué) et correspondent à un été pluvieux, suivant une fin de printemps ou un début d'été sec. Un arrêt, ou au moins une forte diminution, de la croissance, existe après la poussée végétative se produisant au début de chaque printemps (Zhang, 1987), mais ceci semble insuffisant pour provoquer la formation d'un faux cerne. Ainsi une année sans sécheresse estivale, comme celle de 1977, ne présente pas de faux cerne bien que ce soit une année avec une forte croissance. D'après notre étude, c'est bien une période de sécheresse en début d'été suivie d'une période assez pluvieuse qui est nécessaire pour la formation du faux cerne.

En conclusion, Quercus ilex peut tirer parti des variations climatiques interannuelles, en particulier par l'utilisation des précipitations de la fin de l'été ou du début de l'automne. Toutefois, cette valorisation reste faible par rapport à d'autres espèces dans des conditions climatiques semblables. II faut se rappeler néanmoins que les résultats obtenus ici portent sur un nombre assez limité d'échantillons et une série chronologique courte, ce qui doit inciter à une certaine prudence dans leur interprétation biologique et leur généralisation.

\section{REMERCIEMENTS}

Ce travail a reçu l'aide de nombreuses personnes et plus particulièrement de $F$ SerreBachet et J Guiot du Laboratoire de botanique historique et palynologie de la Faculté des sciences et techniques de Saint-Jérôme (Marseille), ainsi que celle de M Grandjanny, JD Lebreton, M Maistre, $P$ Perret et M Roux du CEPE Louis Emberger; nous les en remercions.

Le présent travail est inclus dans un programme soutenu par la CEE et le SRTIE.

\section{RÉFÉRENCES}

Aschmann H (1973) Distribution and peculiarity of mediterranean ecosystems. In: Mediterra- 
nean type ecosystems. Origin and Structure (Di Castri, Mooney, eds). Springer, NewYork, Ecological Studies 7, 11-19

Aschmann $H$ (1984) A restrictive definition of mediterranean climates. Bull Soc Bot Fr Actual Bot 131 (2/3/4), 21-30

Aussenac G, Granier A (1978) Quelques résultats de cinétique journalière du potentiel de sève chez les arbres forestiers. Ann Sci For 35, 19-32

Aussenac G, Valette JC (1982) Comportement hydrique estival de Cedrus atlantica Manetti, Quercus ilex $L$ et Quercus pubescens Willd et de divers pins dans le Mont Ventoux. Ann Sci For 39, 41-62

Box GFP, Jenkins GM (1970) Time series analysis: forecasting and control. Revised edition (1976). Hoden-Day, San francisco

Bronwer R (1977) Root functionning. In: Environmental effects on crop physiology (Landsberg, Cutting, eds) Acad Press, Londres, 229-245

Champagnat $P$ (1983a) Quelques particularités biologiques des arbres. Bull Soc Bot Fr 130, Actual Bot (2), 11-20

Champagnat $P$ (1983b) Quelques réflexions sur la dormance des bourgeons des végétaux ligneux. Physiol Vég 21, 607-618

Daget $P$ (1980) Un élément actuel de la caractérisation du monde méditerranéen : le climat. Naturalia monspeliensa, $n^{\circ} \mathrm{HS}, 101-$ 126

Debussche M, Escarré J (1983) Carte des isohyètes interannuelles dans le Montpelliérais. Document établi pour la série 1950-1979. CNRS-CEPEI Emberger, Montpellier

Eckardt FE (1977) Physiological behaviour in relation to the environment: $A$ comparison between a crop and various types of natural vegetation. In: Environmental effects on crop physiology (Landsberg, Cutting, eds). Acad Press, Londres, 157-171
Emberger $L$ (1955) Une classification biogéographique des climats. Recent Trav Lab Bot Géol Zool Fac Sci, Montpellier, Bot 7, 3-43

Fritts HC (1976) Tree ring and climate. Acad Press, New York

Guiot J (1981) Analyse mathématique de données géographiques. Application à la dendroclimatologie. Thèse, Univ Catholique de Louvain, Fac Sci, Louvain-la-Neuve

Guiot J, Tessier L, Serre-Bachet F (1982) Application de la modélisation ARMA en dendroclimatologie. C $R$ Séances Acad Sci Paris 294, 133-136

Martin P (de) (1974) Analyse des cernes : Dendrochronologie et dendroclimatologie. Masson, Paris

Pons A, Vernet L (1971) Une synthèse nouvelle de l'histoire du Chêne vert (Quercus ilex $L$ ). Bull Soc Bot Fr 118, 841-850

Quézel P (1985) Définition of the Mediterranean region and the origin of its flora. In: Plant conservation in the Mediterranean area (Gomez-Campo, ed). Geobotany 7, 9-24

Serre $F(1980)$ De l'utilité du relevé des caractéristiques des cernes ou "skeleton plot" en dendroclimatologie. Rev Archéométrie 4, 2124

Tessier L (1984) Dendroclimatologie et écologie de Pinus silvestris $L$ et Quercus pubescens Willd dans le Sud-Est de la France. Thèse doctorat d'État. Univ d'Aix-Marseille III

Venet $J$ (1974) Identification et classement des bois français. ENGREF, Nancy

Wendland WM (1975) An objective method to identify missing or false rings. Tree-Ring Bull $35,41-48$

Zhang SH (1987) Contribution à l'étude de la croissance en diamètre du Chêne vert (Quercus ilex $L$ ) en relation avec le climat. Thèse de doctorat. Univ Sci Tech du Languedoc, Montpellier 